\title{
A Biometric Finger Knuckle-Print Pattern Recognition for SURF using Spatial Filters
}

\author{
Utkrsh Kumar \\ Computer Science \& Engineering \\ Oriental Institute of Science \& Technology \\ Bhopal, Madhya Pradesh, India
}

\author{
Sandeep Monga \\ Computer Science \& Engineering \\ Oriental Institute of Science \& Technology \\ Bhopal, Madhya Pradesh, India
}

\begin{abstract}
Biometric is termed as an instinctive recognition technique of a person relied on the extracted physical or behavioural features. Nowadays, consideration of this technique depends on various factors. Finger knuckles can be played as a strong contender among competent features used in biometric system due to its characteristics. Fundamentally, surface of finger knuckle is considered as a unique shape created on the finger joints at their back region of the hands. Here the system pertains to middle joint of the fingers with outer surface for finger knuckle print based authentication system. System uses spatial digital filtration for speeded up robust features (SURF) extraction that increases the precision rate for better authentication. Spatial filter can directly work with pixel image at each point $(\mathrm{x}, \mathrm{y})$. The result is the sum of products of the mask coefficients with the corresponding pixels directly under the mask. It is process of non linear filtration that operates on neighbourhoods and the mechanics of sliding a mask past an image are the same as was just outlined. The proposed system is able to acquire high level of accuracy with minimal error rate.
\end{abstract}

\section{Keywords}

Knuckle Print Authentication, Biometric System, Feature Extraction, Spatial Filter, SURF.

\section{INTRODUCTION}

A finger has certain joints and the highest feature density joint is middle joint of upper surface. The existence of this joint generates shrinks on the outer area of the skin, which forms a cutaneous shape comprising of lines, wrinkles, shapes, etc. So, the shape or pattern formed at the joint of PIP on the finger is taken as the finger knuckle print. Disparate to the finger prints, knuckle patterns are hard to scuffle due to its distillates on the inside surface of the region. Characteristics acquired by the knuckle prints are tremendously inimitable which can be an ideal option for a biometric system. Fig. 1 represents the finger knuckle joints and their region names. As per the representation; system is intended to acquire Proximal Interphalangeal Joint (PIP) for Knuckle print based authentication system.

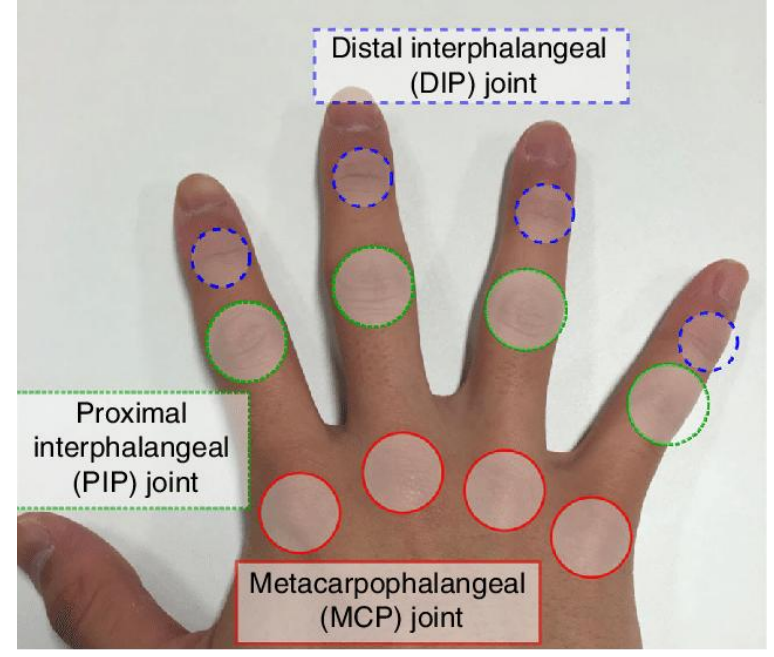

Fig 1: Free Space Detection [1]

\section{RELATED WORKS}

Amine AMRAOUI et al. proposed a concept of Compound Local Binary Patterns (CLBP) where patterns are extracted by encoding all corresponding to a neighbor of local neighborhood in order to construct the compound and robust feature. System uses PolyU database for storing binary templates that intended to compare while authentication [1]. Wafa El-Tarhouni et al. proposed a system which is based on Local Binary Patterns that measures local binaries value of knuckle print texture and compare with the threshold value. Dynamic Threshold Completed Local Binary Patterns dTCLBP technique employs only sign and magnitude components, where the sign component is the same as the original LBP [2]. Walairach Nunsong et al. proposed a system which is based on Fractal Dimension using Gabor filter. Feature extraction is based on Gabor filter grabbed through eight orientations of knuckle print. It covers the upper surface of the finger knuckle skin patterns that possess wrinkles of tilted knuckle. System possesses $96.49 \%$ of accuracy that is an average precision rate of right index, right middle, left index and left middle finger [3]. E. O. Rodrigues et al. proposed a system which is based on Sobel filter and Similarity measures. Sobel is an edge detection technique that finding edges or lines from finger knuckle and preprocess for noise reduction if it contains.

System is intended to capture the knuckle print from folded knuckle that forms less creases or principal lines which results low feature extraction [4]. Farzam Kharaji Nezhadian et al. proposed a system which is based on inner knuckle print instead of outer one. In this system the inner surface of finger has been used authentication that may possess less features or texture. Gabor wavelet filter has been used for enhancing the 
inner knuckle print and feature extraction has been done using K-Nearest neighbor fuzzy classifier [5]. Arulalan. V et al. proposed a combinational biometric system of two traits Iris and Finger knuckle print; it means that the score will be captured on the basis of two distinct features. Here Haar Wavelet has been used for Iris feature extraction and Linear Discriminant Analysis for finger knuckle print [6]. Jooyoung Kim et al. proposed a finger knuckle print system based on difference between the images with respect to the Euclidean distance comparison. Here the difference image operation has been performed to enhance the knuckle-print lines on the image matrix [7]. Amine AMRAOUI et al. proposed a concept of Compound Local Binary Patterns (CLBP) where patterns are extracted by encoding all corresponding to a neighbor of local neighborhood in order to construct the compound and robust feature. System uses PolyU database for storing binary templates that intended to compare while authentication. Author used distance based classifier for reducing the computational time. The accuracy trails to 98.71 $\%$ for authenticating the legitimate and illegitimate users [8].

\section{PROBLEM STATEMENT}

There are some flaws in previously proposed systems that pertain precision rate of the systems because local binary patterns easily affected by the bright luminance and features get distorted. Enhancing image may suffer distortion that directly connected to the feature which knuckle image contains. Combining two biometric systems is not a solution or enhancement towards knuckle print based authentication system or knuckle feature extraction.

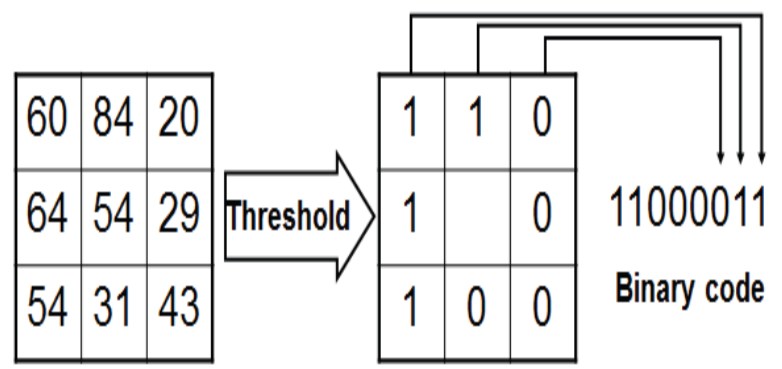

Fig 2: Local Binary Patterns [3]

Previously proposed system is based on Local Binary Patterns and PolyU database. Since the method uses predefined algorithm which limits the practical implementation of system as slight illumination can affect the accuracy of knuckle print scanner. Binary values may differ on different light occurrence that resulted inaccurate precision. If a single false acceptance made among 1000 of users then system get failed to provide ideal authentication system. Figure 2 shows the binary code representation of an image. Binary code based template matching affects the accuracy directly because of lightness or luminance and does not work with poor image. Local binary pattern has been used by several researchers but do not met desirable accuracy. It is a widely used effect in graphics software, typically to reduce image noise. Less pattern details extracted that possesses less accuracy rate.

\section{PROPOSED WORK}

The proposed system is able to provide better authentication approach towards knuckle print. It acquires user's knuckle in the form of frame and later processes it for feature extraction that can be compared with the stored template. The true acceptance rate of proposed method is bit higher as compare to the earlier implemented system with minimal error rate.
Here the system is based on spatial filter for extracting speeded up robust features. Spatial filter is able to extract key features from an image with high level of precision without distorting image sensitivities. It is a digital image filtration technique through which speeded up robust features can be determined. In computer vision, speeded up robust features (SURF) is a patented local feature detector and descriptor. It can be used for tasks such as object recognition, image registration, classification or $3 \mathrm{D}$ reconstruction. Spatial filtering is an image processing technique to change the pixel intensity according to the intensity of the neighboring pixels.

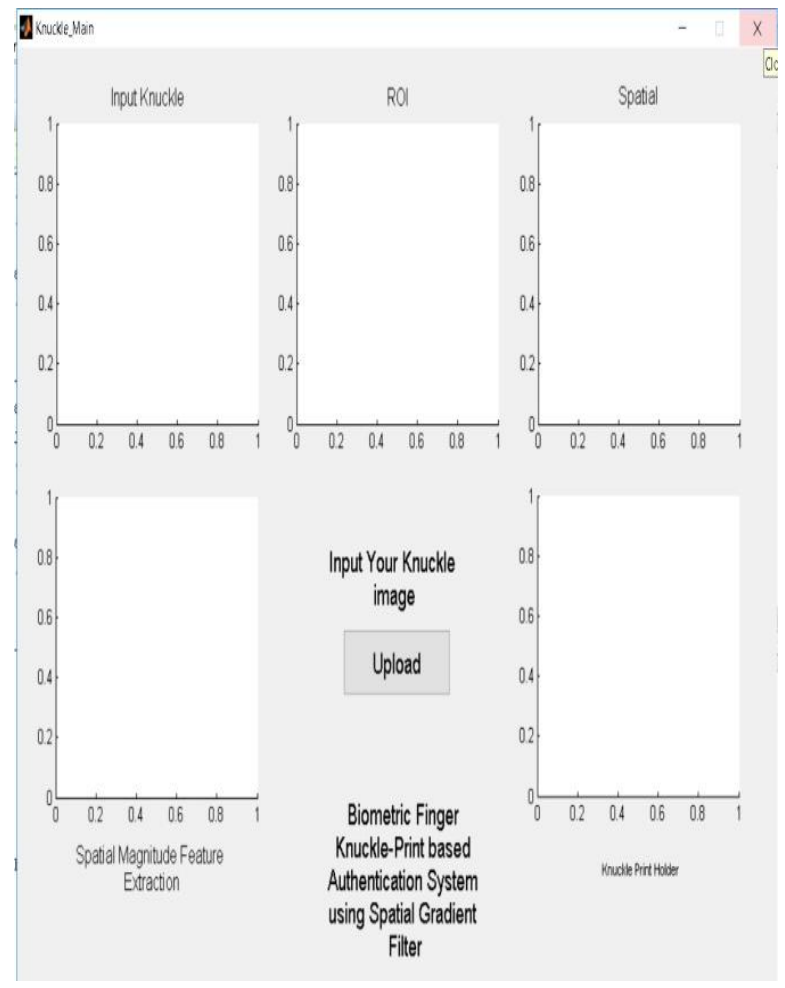

Fig 3: Proposed System GUI

Fig. 3 shows the graphical user interface for uploading or capturing Iris image for further proceeding. System uses spatial filter instead of Canny edge detection that resulting better feature extraction. Spatial filter is a method of advanced digital image filtering technique for extracting information from a digital image. User is required to process the image for feature extraction and template creation, once the template has been created, it will be stored in the database for future matching. If a user input an Iris image that belongs to the database, it will started capturing ROI (region of interest) and proceed for feature extraction and once the feature has been extracted it will started matching key features and if key feature is greater than the threshold value, threshold value is a value where the condition has been applied for decision making, if it satisfies the threshold then user will be authenticated and details will become appeared accordingly. But if computed key feature is less than the threshold value then user will get denied. As per the scientific law if $20 \%$ key points get matched then it belongs to that person. So that is why the threshold value is set to $20 \%$ of key features or points found. 


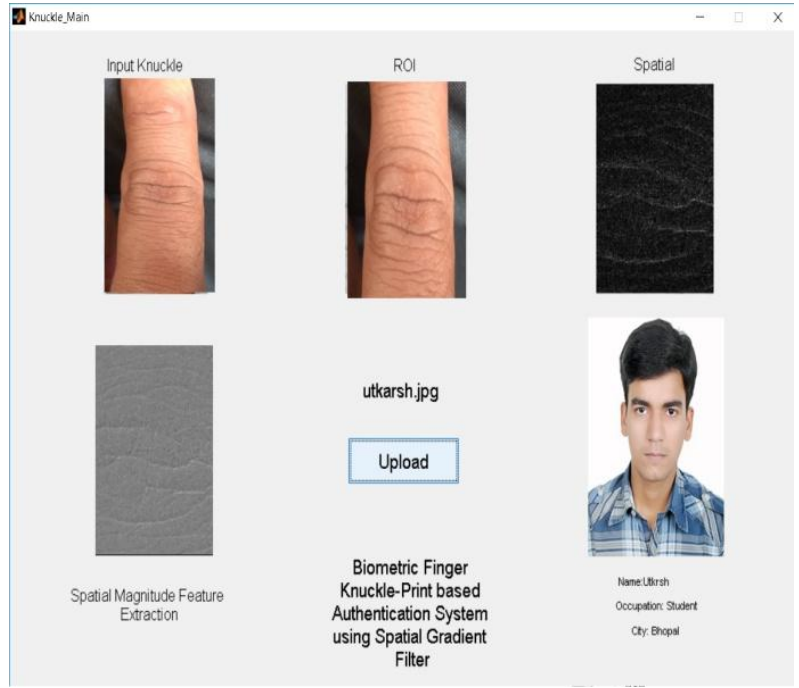

Fig 4: Success Authentication

System is capable to return true positive results with minimal false or fake results. System has been tested with many enrollments and system observed as effective biometric authentication system.
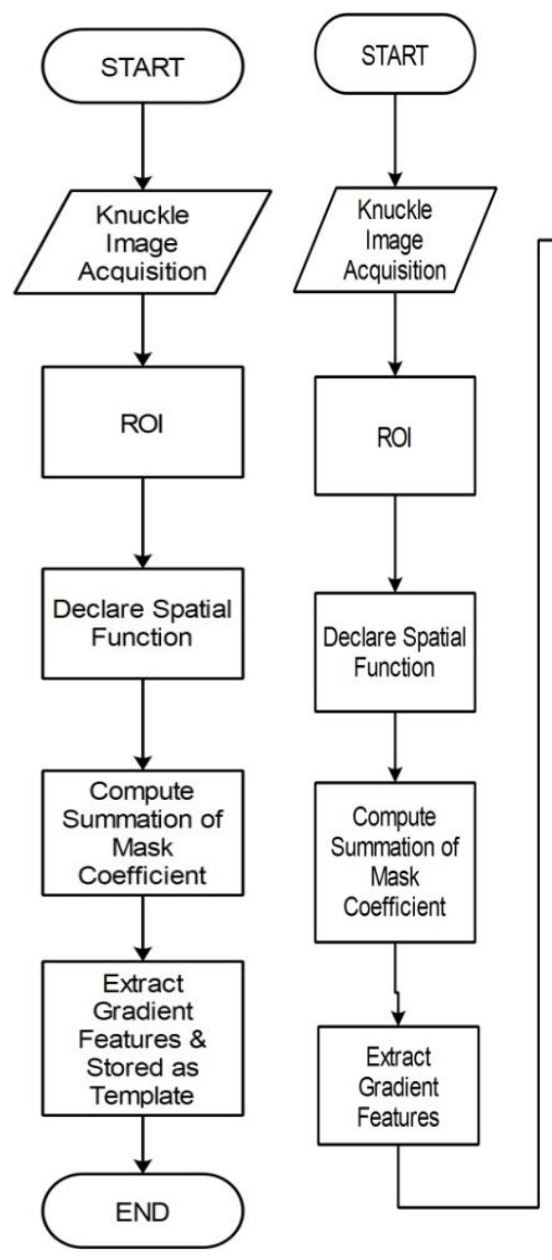

a)

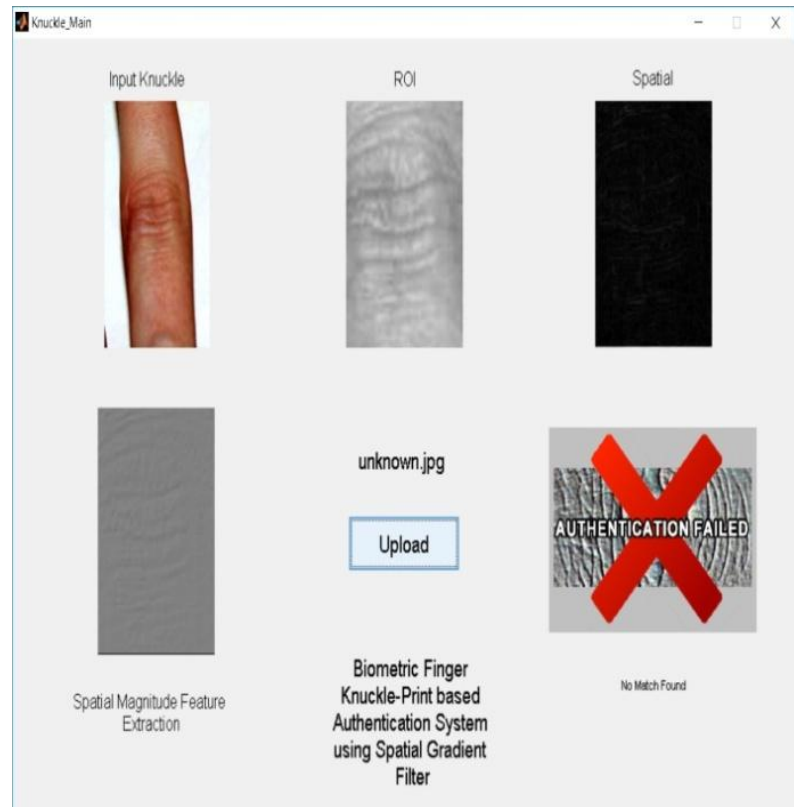

Fig 5: Denied Authentication

Fig 6: Flow Charts for a) Feature Extraction \& b) Feature Matching 
In the training phase it is required to create feature templates of knuckle images as per the authentication being tested. First of all a knuckle image has been acquired to obtain the edge detection by applying spatial filter tool. Once the gradient features have been extracted the feature has been declared in the form of texture, these textures later stored as template for future matching. The extraction process is similar to the training process except template matching. Once the feature has been extracted, it compared with the template that stored previously. If it is greater than the threshold data points then it will fetch the user details else it will deny the user. Spatial filtering term is the filtering operations that are performed directly on the pixels of an image.

\section{PROPOSED METHODOLOGY}

\subsection{Spatial Magnitude Algorithm}

Require: $\mathrm{K}_{\mathrm{i}}$ as 2 dimensional image array, $\mathrm{f}(x, y)$ as spatial function, Horizontal gradient mask $\mathrm{G}_{\mathrm{x}}$, Vertical gradient mask $\mathrm{G}_{\mathrm{y}}$, threshold $\mathrm{T}$, Absolute magnitude $\nabla f$, template $\mathrm{F}_{\mathrm{t}}$

INPUT: $\mathrm{K}_{\mathrm{i}} \leftarrow 2$ dimensional image arrays.

OUTPUT: Filtered Image Derivatives

Step 1: Acquire the input knuckle image as $K_{i}$.

Step 2: Function Spatial (A) set to $\mathrm{f}(x, y)$

Step 3: Initially set function $\mathrm{f}(x, y)$ to $\mathrm{w}(0,0)$

Step 4: $\mathrm{w}(-1,1), \mathrm{w}(-1,0) \ldots \mathrm{w}(-n, m)$ are corresponding neighboring pixel with mask coefficients

Step 5: Compute the sum of mask coefficients -

$\mathrm{f}(x, y)=\mathrm{w}(-1,-1) \mathrm{f}(x-1, y-1)+\mathrm{w}(-1,0) \mathrm{f}(x-1, y)+\mathrm{w}(-1,1) \mathrm{f}(x-$ $1, y+1) \ldots \ldots+\mathrm{w}(n, m) \mathrm{f}(x+a, y+b) \mathrm{f}(x, y)=\sum_{i=1}^{n m} w_{i} f_{i}$

Step 6: Unsharp Masking-

$(x, y)=f(x, y)-\bar{f}(x, y)$

$f_{s}(x, y)$ denotes the sharpened image obtained by unsharp masking, $f(x, y)$ is a blurred version of $\bar{f}(x, y)$

Step 7: Compute first derivatives using the magnitude of the gradient

$$
\nabla f=\left[\begin{array}{l}
G_{x} \\
G_{y}
\end{array}\right]=\left[\begin{array}{l}
\frac{d f}{d x} \\
\frac{d f}{d y}
\end{array}\right]
$$

Step 8: The magnitude of the vector is computed as -

$$
\nabla f=\sqrt{G_{x}^{2}+G_{y}^{2}} \approx\left|G_{x}\right|+\left|G_{y}\right|
$$

Step 9: Store filtered magnitude as template for enrollment as $\mathrm{F}_{\mathrm{t}} /$ compare with the templates for authentication

\section{Step 10: if $F_{t}>T$ then}

Authenticate user;

else

Denying user;

end else

end if

Step 11: End

Here the input is a 2 dimensional image array and output is gradient filtered image derivatives. First of all a knuckle image is to be acquired for further process and once it has been acquired then spatial function will be applied for calculating gradient values. Once the gradient value has been calculated in $\mathrm{x}$ and $\mathrm{y}$ axis the gradient magnitude is to be calculated. A feature has been extracted as key points that can be stored in database for future matching. While performing authentication, feature extraction process is similar to the enrollment process. If extracted feature is greater than the threshold value then it will authenticate the user otherwise deny. Similarities are based on key point extracted from input image and the image stored in the database.

\section{RESULT ANALYSIS}

The result has been analyzed on the basis of true acceptance, true rejection, false acceptance and false rejection. Here the total number of 25 true knuckle image tested that belongs to stored templates. All true users get accessed with true authentication and there is no true rejection at all, similarly 25 false users are also tested for error computation. There are no false users get accessed, all are denied by the system.

Table 1 Result Analysis

\begin{tabular}{|c|c|c|c|}
\hline Holders & $\begin{array}{c}\text { Key points } \\
\text { Found }\end{array}$ & $\begin{array}{c}\text { Key points } \\
\text { Matched }\end{array}$ & Score \% \\
\hline 1 & 1070 & 1070 & $100 \%$ \\
\hline 2 & 302 & 302 & $100 \%$ \\
\hline 3 & 1211 & 1186 & $97.93 \%$ \\
\hline 4 & 589 & 587 & $99.66 \%$ \\
\hline 5 & 785 & 741 & $94.39 \%$ \\
\hline 6 & 799 & 799 & $100 \%$ \\
\hline 7 & 877 & 877 & $100 \%$ \\
\hline 8 & 1040 & 1040 & $100 \%$ \\
\hline 9 & 691 & 680 & $98.40 \%$ \\
\hline 10 & 547 & 547 & $100 \%$ \\
\hline & & & \\
\hline
\end{tabular}

Table 1 represent the key points extracted from input image and key points that are stored in database. Accuracy is computed on the basis of score level and matching key points. 
Table 2 Result Comparison

\begin{tabular}{|l|l|}
\hline & Accuracy \\
\hline CLPP [11] & $86.26 \%$ \\
\hline OCLPP [12] & $87.42 \%$ \\
\hline MSLBP [13] & $93.87 \%$ \\
\hline LBP [14] & $98.71 \%$ \\
\hline Proposed & $99.03 \%$ \\
\hline
\end{tabular}

Table 2 represent the comparison over the last decades researches on knuckle print based authentication system. As per the accuracy computed is higher than the previous one. The accuracy of successful key points matching is $99.03 \%$ along with $100 \%$ false rejection rate and true acceptance rate as per the samples tested. The accuracy is calculated over the 10 score levels or key points, the ratio for deriving precision is based on key points found on an image and how many key points matched. There is no false acceptance that shows an ideal system with minimal error rate.

Graph 1 Result Comparison

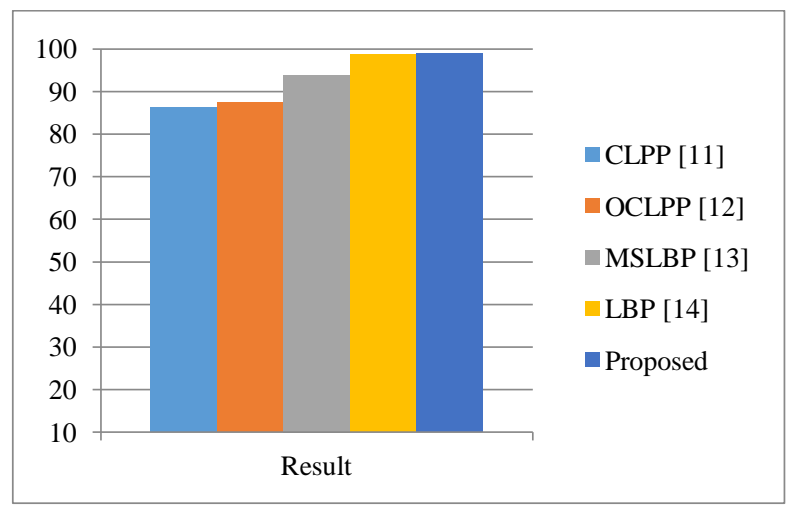

Graph 1 shows the precision comparison among various researches made over biometric knuckle print based authentication system.

\section{CONCLUSION \& FUTURE SCOPE}

Thus the currently proposed system is able to recognize knuckle region along with its features that correctly authenticate the user and rejecting the false on. Precision is calculated over the key points matching scores and error rate is computed as per the false acceptance, false rejection, true acceptance and true rejection. So, the accuracy rate of proposed work is $99.03 \%$ along with $100 \%$ false rejection and true acceptance rate. Spatial filtering technique is liable to acquire the accuracy rate which is bit higher from previous works. Knuckle print based authentication system can be implemented with bit higher precision in future and applicable in various fields of authentication. Knuckle biometric authentication is a touch-less system that allows users to interact without any hygienic disease. This biometric feature cannot be stolen and no unauthorized users get access. This system can be implemented in various fields that may replaces existing one.

\section{REFERENCES}

[1] https://www.researchgate.net/figure/A-taxonomy-offinger-knuckle-joints-Blue-colored-circles-indicatedistal-interphalangeal_fig1_315637217

[2] Neerja Deogaonkar , Harshada Kahar ,Bhagyshri Parab ,Snehal Rajpure , Disha Bhosle, "Biometric Authentication Using Finger Knuckle Print" IOSR Journal of VLSI and Signal Processing (IOSR-JVSP) Volume 6, Issue 1, Ver. I (Jan. -Feb. 2016), PP 55-59.

[3] Amine AMRAOUI*, Youssef FAKHRI and Mounir AIT KERROUM, " Finger Knuckle Print Recognition System using Compound Local Binary Pattern", 3rd International Conference on Electrical and Information Technologies ICEIT'2017, IEEE.

[4] Jooyoung Kim, Kangrok Oh, Andrew Beng-Jin Teoh and Kar-Ann Toh, "Finger-Knuckle-Print for Identity Verification Based on Difference Images" 2016 IEEE 11th Conference on Industrial Electronics and Applications (ICIEA).

[5] Arulalan. V and Dr. K.Suresh Joseph, "Score Level Fusion of Iris and Finger Knuckle Print", 2016 10th International Conference on Intelligent Systems and Control (ISCO), IEEE.

[6] FarzamKharajiNezhadian and Saeid Rashidi, "Innerknuckle-print for human authentication by using ring and middle fingers", ICSPIS 2016, 14-15 Dec. 2016 Amirkabir University of Technology, Tehran, Iran, IEEE.

[7] E. O. Rodrigues, T. M. Porcino, A. Conci and A. C. Silvah, "A simple approach for biometrics: Fingerknuckle prints recognition based on a Sobel filter and similarity measures, " 2016 International Conference on Systems, Signals and Image Processing (IWSSIP), Bratislava, 2016, pp. 1-4.

[8] Wafa El-Tarhouni1, Larbi Boubchir2 and Ahmed Bouridane1, "Finger-Knuckle-Print Recognition Using Dynamic Thresholds Completed Local Binary Pattern Descriptor", 2016 39th International Conference on Telecommunications and Signal Processing (TSP), IEEE.

[9] I. S. Oveisi and M. Modarresi, "A feature level multimodal approach for palmprint and knuckleprint recognition using AdaBoost classifier," 2015 International Conference and Workshop on Computing and Communication (IEMCON), Vancouver, BC, 2015, pp. 1-7.

[10] Steve, https://blogs.mathworks.com/steve/2016/05/16/imagebinarization-new-r2016a-functions/, Published on April $16^{\text {th }}, 2016$.

[11] D. Zhang, and M. S. Kamel, "An analysis of iriscode," IEEE transactions on image processing, vol. 19, no. 2, pp. 522-532, 2010.

[12] S. Agarwal and P. Gupta, "Identification of human through palmprint: A review," International Journal of Advanced Research in Computer Engineering \& Technology (IJARCET), vol. 1, no. 10, pp. pp-19, 2012.

[13] X.-Y. Jing and D. Zhang, "A face and palmprint recognition approach based on discriminant dct feature extraction," IEEE Transactions on Systems, Man, and 
Cybernetics, Part B (Cybernetics), vol. 34, no. 6, pp. 2405-2415, 2004.

[14] D. Zhang, Z. Guo, G. Lu, L. Zhang, and W. Zuo, "An online system of multispectral palmprint verification," IEEE transactions on instrumentation and measurement, vol. 59, no. 2, pp. 480-490, 2010

[15] W. K. Kong, D. Zhang, and W. Li, "Palmprint feature extraction using 2-d gabor filters," Pattern recognition, vol. 36, no. 10, pp. 2339-2347, 2003.

[16] D. I. Devi and B. T. G. Sampantham, "An efficient security system based on gabor feature detector," in Control, Automation, Communication and Energy Conservation, 2009. INCACEC 2009. 2009 International Conference on, pp. 1-6, IEEE, 2009.

[17] W. Li, D. Zhang, and Z. Xu, "Image alignment based on invariant features for palmprint identification," Signal Processing: Image Communication, vol. 18, no. 5, pp. 373-379, 2003.

[18] W. Jia, R.-X. Hu, J. Gui, Y. Zhao, and X.-M. Ren, "Palmprint recognition cross different devices," Sensors, vol. 12, no. 6, pp. 7938-7964, 2012.

[19] D. Zhang, V. Kanhangad, N. Luo, and A. Kumar, "Robust palmprint verification using $2 \mathrm{~d}$ and $3 \mathrm{~d}$ features," Pattern Recognition, vol. 43, no. 1, pp. 358-368, 2010.

[20] K. Krishneswari and S. Arumugam, "A review on palm print verification system," International Journal of Computer Information Systems and Industrial Management Applications (IJCISIM) ISSN, pp. 21507988, 2010.
[21] Z. Guo, W. Zuo, L. Zhang, and D. Zhang, "Palmprint verification using consistent orientation coding," in Image Processing (ICIP), $200916^{\text {th }}$ IEEE International Conference on, pp. 1985-1988, IEEE, 2009.

[22] W. Li, B. Zhang, L. Zhang, and J. Yan, "Principal linebased alignment refinement for palmprint recognition," IEEE Transactions on Systems, Man, and Cybernetics, Part C (Applications and Reviews), vol. 42, no. 6, pp. 1491-1499, 2012.

[23] M. Mu, Q. Ruan, and Y. Shen, "Palmprint recognition based on discriminative local binary patterns statistic feature," in Signal Acquisition and Processing, 2010. ICSAP'10. International Conference on, pp. 193-197, IEEE, 2010.

[24] S.S. Khot, V.A. Mane and K.P. Paradeshi, "Real Time Palm print Identification Technique-Effective Biometric Identification Technique", International Journal of Societal Applications of Computer Science, Vol. 1, Issue 1, November 2012

[25] Wenxin Li, David Zhang and Zhuoqun Xu, "Image alignment based on invariant features for Palm print identification", Signal Processing: Image Communication, Vol. 18, pp. 373-379, 2003.

[26] Wei Jia, Rong-Xiang Hu, Jie Gui, Yang Zhao and XiaoMing Ren, "Palm print Recognition across Different Devices", Sensors, ISSN: 1424-8220, Vol. 12, pp. 7938 7964, 2012.

[27] David Zhang, Vivek Kanhangad, Nan Luo and Ajay Kumar, "Robust Palm print Verification Using 2D and 3D Features", Pattern Recognition, Vol. 43, No. 1, pp. 358-368, January 2010. 